B6 mice $(<0.0001)$, reflecting increased anxiety. There were no differences motor coordination, supporting an anxiety phenotype leading to decreased movement in the open field. On novel object placement testing, there were no differences in time spent with the novel object. Lastly, the forced swim test revealed that $\mathrm{B} 6 . \mathrm{Nba} 2$ mice spent more time immobile than B6 mice $(\mathrm{p}<0.02)$, suggesting a depressive-like behavior in B6. $\mathrm{Nba} 2$ mice.

Conclusions Our data suggest that the B6.Nba2 mouse model expresses a strong anxiety phenotype, a depressive phenotype, but no deficits in spatial memory. This observation warrants further exploration of the B6.Nba2 as a mouse model of NPSLE along with analyses of brain histology and morphology and future evaluations of the relationship between IFN-, autoantibody levels and neurological disease manifestations.

Funding Source(s): None

\section{EVALUATION OF PSYCHOMETRIC PROPERTIES OF THE PATIENT-REPORTED OUTCOMES MEASUREMENT INFORMATION SYSTEM PHYSICAL FUNCTION 10-ITEM SHORT FORM IN SYSTEMIC LUPUS ERYTHEMATOSUS}

'Zara Izadi* ${ }^{2}$ Julia Kay, ${ }^{3}$ Gabriela Schmajuk, ${ }^{4}$ Jinoos Yazdany. ${ }^{1}$ University of California, San Francisco; ${ }^{2}$ Division of Rheumatology, Department of Medicine, University of California, San Francisco; ${ }^{3}$ University of California, San Francisco; San Francisco VA Medical Center; ${ }^{4}$ UC San Francisco

\subsection{6/lupus-2019-Ism.108}

Background While the PROMIS (Patient-Reported Outcomes Measurement Information System) physical function short form 10a (PF10a) is both practical and acceptable for implementation in routine clinical practice, its psychometric properties have not been evaluated in Systemic Lupus Erythematosus (SLE). We examined the validity and responsiveness of PF10a in SLE among a racially/ethnically diverse clinic population and developed estimates of the minimally important difference (MID).

Methods Data were derived from electronic health records for all SLE patients seen in a university-based rheumatology clinic between 2013 and 2018. We evaluated the PF10as floor and ceiling effects among different racial/ethnic groups. Construct validity was assessed by examining Spearmans correlation coefficients between the PF10a and other patient-reported (pain (scale 0-10) and pain visual analogue scale (VAS) (scale 0100)), physician-reported (SLE disease activity index (SLEDAI)) and laboratory (erythrocyte sedimentation rate (ESR)) measures. Known-group validity was assessed by evaluating effect size (Cohens d) between categories of pain (no pain vs. moderate-severe pain). We used standardized response means to examine the responsiveness of the PF10a to longitudinal changes in pain and SLEDAI. MID was estimated using distribution based and anchor-based methods.

Results We included 612 patients in cross-sectional analyses of validity and 462 patients in longitudinal analyses of responsiveness. Mean age was $40.5 \pm 14.6,87 \%$ were female and $32 \%$ Caucasian. The PF10a had ceiling effects above the commonly accepted criteria of $15 \%$ among Caucasian (23\%), Asian (23\%) and Other (17\%) race/ethnicities, and no floor effects. Construct validity analyses showed strong correlations $(=0.66, \mathrm{p}<0.05)$ with pain VAS, moderate correlations $(=0.58, \mathrm{p}<0.05)$ with pain, and weak correlations with ESR $(=0.25, \mathrm{p}<0.05)$ and SLEDAI $(=0.16, \mathrm{NS})$. Known-group validity analyses showed large differences among pain groups
(Cohens $\mathrm{d}=1.49, \mathrm{p}<0.05)$. The PF10a was responsive to improvements in pain $(S R M=0.5)$ and SLEDAI $(0.49)$, but less so to deteriorations in pain $(S R M=-0.42)$ or SLEDAI (SRM=-0.24). Distribution-based MIDs were +8 for improvement and -7 for deterioration. Anchor-based MIDs were +2 for improvement, -3 for deterioration with pain as anchor and +5 for improvement, -5 for deterioration with SLEDAI as anchor.

Conclusions Although the PF10a showed some ceiling effects, it had good validity in this young racially/ethnically diverse sample with SLE. The PF10a was responsive to improvements in pain and disease activity. The anchor-based MIDs appear to be similar to those reported for PF10a in rheumatoid arthritis. This information supports the use of the PF10a in SLE and provides important information to facilitate interpretation of scores.

Funding Source(s): UCSF PREMIER

\section{DERMAL LYMPHATIC CHARACTERIZATION AND PHOTOSENSITIVITY IN THE MRL/LPR LUPUS MODEL}

${ }^{1}$ Noa Schwartz*, ${ }^{1}$ Susan Chyou, ${ }^{2}$ Thomas Morgan, ${ }^{3}$ William D Shipman, ${ }^{4}$ Theresa T Lu. ${ }^{1}$ Hospital for Special Surgery; ${ }^{2}$ Columbia University; ${ }^{3}$ Hospital for Special Surgery; ${ }^{4}$ Autoimmunity and Inflammation Program and Pediatric Rheumatology, Hospital for Special Surgery

10.1136/lupus-2019-Ism.109

Background Proper function of lymphatic vessels is needed to limit the magnitude and duration of tissue inflammation, and in direct regulation of immune cell activity. Inflammatory states such as rheumatoid arthritis and psoriasis are associated with lymphatic dysfunction, but lymphatics in lupus models have not been well characterized. SLE patients are photosensitive, developing inflammatory skin lesions upon exposure to even ambient ultraviolet radiation (UVR). We hypothesized that lymphatic dysfunction may contribute to photosensitivity in lupus.

Methods MRL/MpJ-Faslpr/lpr (lpr) mice and age-/sex-matched MRL controls were evaluated at baseline, 1 day, 1 week and 1 month after exposure to $2000-2500 \mathrm{~J} / \mathrm{m}^{2}$ of UVB radiation. Lymphatic function was assessed with an intradermal injection of $1 \mu \mathrm{L}$ of $2 \%$ evans blue (EB) to the ear, followed by measurement of $\mathrm{EB}$ concentration in the draining lymph node (dLN) 1 min later. Flow cytometry of ears and dLN allowed quantification of resident cell populations. KruskalWallis test, followed by Dunns test for multiple comparisons were used to compare the groups; data is presented as mean \pm SE, with a two-tailed p-value of $<0.05$ considered significant.

Results At baseline, the effective flow of EB, calculated as EB concentration in the dLN, per ear lymphatic endothelial cell (LEC) number, is comparable between the 2 groups. Following UVR, in both lpr and control mice, there is an increase in the effective flow of $\mathrm{EB}$ to the $\mathrm{dLN}$, that starts as early as 1 day, and continues to increase at 1 week, in similar levels between the 2 groups. At 1 month post-UVR, however, the flow continues to improve in the MRL control mice, but there is no similar improvement in the lpr mice. In fact, while at baseline, 1 day and 1 week the mean relative lymphatic flow in the lpr mice ranges from 1.6-, 2.5- and 0.9- fold of controls, respectively, at 1 month the effective flow in the $1 \mathrm{pr}$ mice is only $5 \%$ that of controls $(p=0.007)$. 